\title{
Sphere Wakes in Still Surroundings at Intermediate Reynolds Numbers
}

\author{
J.-S. Wu* and G. M. Faeth† \\ University of Michigan, Ann Arbor, Michigan 48109
}

\begin{abstract}
The wakes of spheres in a still environment were studied for sphere Reynolds numbers $R e$ in the range 30-4000. The experiments consisted of towed spheres in quiescent baths of glycerin and water mixtures. Measurements included dye traces illuminated by a laser light sheet for visualization and laser velocimetry for streamwise velocities. The recirculation region on the downstream side of the sphere was stable and symmetric for $R e<200$, stable and unsymmetric for $200 \leq R e \leq 280$, and unstable with vortex shedding for $R e \geq 280$. Three wake regions were identified: a fast-decay region that was observed only when vortex shedding was present, followed in succession by turbulent and laminar wake regions. Vortex shedding increased the distance to the onset of the turbulent wake region by an order of magnitude due to the presence of the fast-decay wake region. Mean velocities within the turbulent and laminar wake regions scaled according to classical similarity theories, with transition between these regions at conditions where their estimates of mean streamwise velocities along the axis were the same: this occurred at a local wake Reynolds number $R e_{w} \approx 10$. Within the turbulent wake region, turbulence intensities along the axis were roughly $85 \%$ for $R e_{w}>70$; however, as the onset of the laminar wake region was approached, turbulence intensities along the axis were proportional to $R e_{w}{ }^{7 / 4}$, which is consistent with scaling proposed earlier for the final decay period of axisymmetric wakes.
\end{abstract}

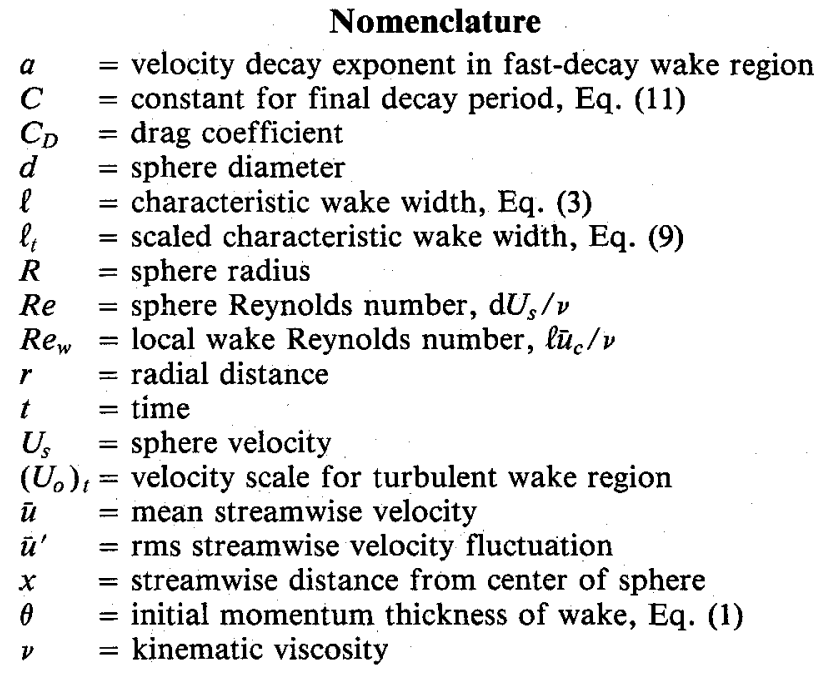

Subscripts

$c=$ centerline value

$\mathrm{cm}=$ uncorrected centerline value

$o \quad=$ virtual origin condition

tr $=$ transition from turbulent to laminar wake

$\infty \quad=$ ambient condition

\section{Introduction}

$\mathbf{T}$ HE flow associated with spheres has attracted attention due to numerous applications, e.g., dispersed particleladen flows, sprays, and rainstorms, among others. Recent work on the production of turbulence by dispersed phases, ${ }^{1,2}$ however, has shown the need for more information about the structure of sphere wakes at the intermediate sphere Reynolds

Received Jan. 28, 1992; revision received Nov. 10, 1992; accepted for publication Nov. 16, 1992. Copyright (C) 1992 by the American Institute of Aeronautics and Astronautics, Inc. All rights reserved.

* Graduate Student Research Assistant, Department of Aerospace Engineering.

$\uparrow$ Professor, Department of Aerospace Engineering. Fellow AIAA. numbers $\left(10<\operatorname{Re}<10^{3}\right)$ that often are encountered for drops and particles in sprays and other dispersed flows. Particularly important issues are the effects of turbulence and vortex shedding from the sphere on wake structure, and the range of conditions for which such effects are observed. Motivated by these observations, the objective of the present investigation was to measure flow properties near spheres at intermediate Reynolds numbers, with particular emphasis on properties within the wake.

Early studies of the flow associated with spheres at intermediate Reynolds numbers are discussed in the extensive series of review articles by Torobin and Gauvin, ${ }^{3}$ and references cited therein. Initial work emphasized drag and flow properties near the sphere, with later experimental and computational work along these lines reported by Pruppacher et al., ${ }^{4}$ Rimon and Cheng, ${ }^{5}$ and Roos and Willmarth. ${ }^{6}$ Subsequently, several studies focused on the near wake of the sphere, including the nature of the recirculation zone behind the sphere and the characteristics of vortex shedding from this region. ${ }^{7-16}$ These results established that a recirculation zone begins to form at a $R e$ of roughly ten, that this zone grows in size as the $R e$ increases, and that vortex shedding from the sphere into the wake begins at roughly $R e=300$ and continues to affect nearwake properties at higher values of $R e$ in the intermediate Reynolds-number regime. Within the intermediate Reynoldsnumber regime, the configuration of vortices shed from the sphere involves closed-end double-helix vortex tubes unwinding from a cylindrical vortex sheet around the periphery of the sphere, giving the appearance of a vortex street passing into the sphere wake when viewed in cross section. ${ }^{15,16}$ These results were obtained from flow visualization, however, so there is little quantitative information available about the effect of vortex shedding on wake properties.

Existing quantitative information about sphere wakes is limited largely to the turbulent wake (see Refs. 17-22 and references cited therein). This involved measurements in the wakes of various axisymmetric objects far enough downstream so that wake properties did not exhibit the periodic behavior associated with vortex shedding and were controlled by the drag rather than the shape of the object. These measurements generally were completed for object (sphere) Reynolds numbers on the order of $10^{4}$ or greater, with local wake Reynolds numbers on the order of $10^{2}$ or greater. Thus, the properties of 
wakes for intermediate Reynolds numbers, or even whether turbulent wakes are present for such conditions, are unknown in spite of the importance of this Reynolds-number range for dispersed multiphase flows. ${ }^{1,2}$

The present investigation was undertaken to provide new information about the structure of the flow near spheres at intermediate sphere Reynolds numbers. This included visualization of the flow near the spheres to provide information about the recirculation zone and vortex shedding into the wake, measurements of mean streamwise velocities both near the spheres and in their wakes, and measurements of rms velocity fluctuations along the flow axis, extending to conditions where the wakes were laminar. The experiments involved spheres towed through quiescent water and glycerol mixtures to achieve sphere Reynolds numbers in the range $30-4000$. Within both the turbulent and laminar portions of the wakes, velocity measurements were compared with classical similarity correlations and correlations of turbulence properties in wakes, ${ }^{18-27}$ to help define conditions for the transition between these regimes and the scaling of turbulence properties at intermediate wake Reynolds numbers.

\section{Experimental Methods}

\section{Apparatus}

A sketch of the test apparatus appears in Fig. 1. The experiment involved traversing a sphere through a still liquid bath and observing flow properties at the center of the bath. The liquid bath was filled to a depth of $875 \mathrm{~mm}$ within a windowed tank $(415 \times 535 \times 910 \mathrm{~mm})$. The sides and bottom of the tank were covered with insulation (not shown in Fig. 1) to minimize natural convection disturbances, aside from small openings needed for optical access. The viscosity of the bath liquid was varied so that a range of sphere Reynolds numbers could be considered using a sphere of fixed size and a modest range of velocities.

The test sphere was a $10-\mathrm{mm}$-diam plastic ball (polished polycarb ball, $\pm 50 \mu \mathrm{m}$ radius tolerance, sphericity within 50 $\mu \mathrm{m}$, surface roughness less than $16 \mu \mathrm{m}$ ). The sphere was mounted on a $125-\mu \mathrm{m}$-diam stainless-steel wire which passed horizontally through its center and was sealed with epoxy. The wire was mounted in tension between two struts that could be traversed down the corners of the tank. Tests with no sphere present showed that disturbances from the wire support system were negligible in comparison to background disturbances.

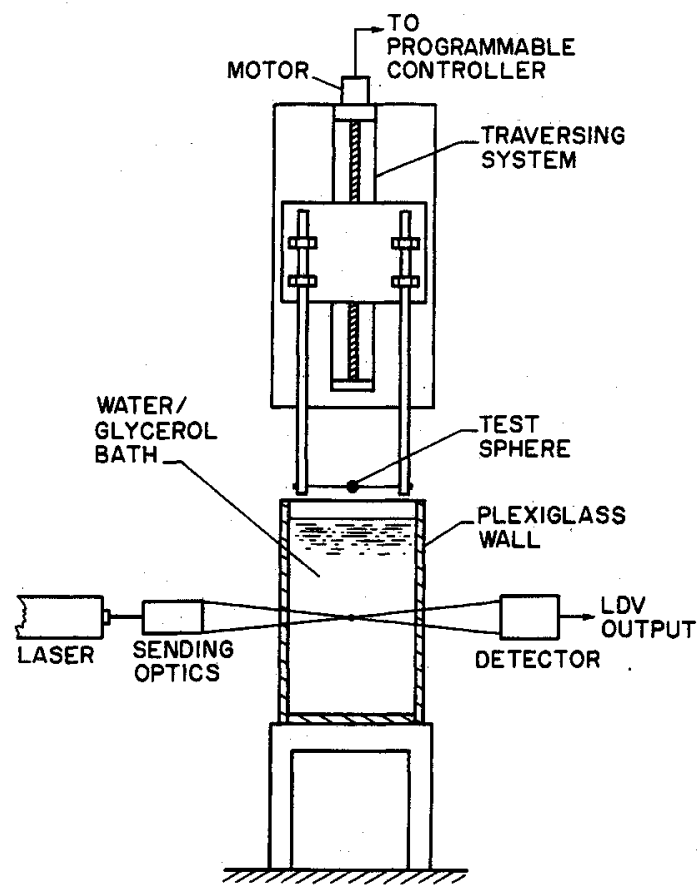

Fig. 1 Sketch of the experimental apparatus.
Additionally, the arrangement was sufficiently rigid so that sphere vibrations during a traverse could not be detected:

The traversing system involved a counterbalanced mounting plate, which could move along a linear bearing system, to support the struts. The motion of the plate was controlled by a stepping motor driven linear positioner (Daedal, Model 0082686 single-axis positioner). The positioner was programmed so that it accelerated to the appropriate sphere velocity (147$476 \mathrm{~mm} / \mathrm{s}$ ) in the first $150 \mathrm{~mm}$ of travel, just prior to entering the bath. The test velocity was maintained for the next $700 \mathrm{~mm}$ ( $300 \mathrm{~mm}$ beyond the measuring station) before decelerating to a stop at the bottom of the tank in the last $150 \mathrm{~mm}$ of travel. The arrangement provided test sphere velocities with an accuracy of $1 \%$, based on the position indicator of the traversing system, with velocity variations within this range for the constant speed portion of the traverse.

\section{Instrumentation}

Flow Visualization

Light sheet illuminated dye traces were used to observe the recirculation region behind the sphere and the vortex shedding process, similar to past work..$^{6-16}$ This was done by painting a liquid soluble dye (Higgins drawing ink no. 4085) near the forward stagnation point of the sphere and photographing the dye trace after illumination by a vertical light sheet passing through the axis of the flow. The light sheet was formed by focusing the 488-nm line of an argon-ion laser with a spherical lens to yield a waist diameter of $200 \mu \mathrm{m}$, and then spreading the beam with a cylindrical lens to illuminate a $300-\mathrm{mm}$ section along the axis of motion. The resulting dye pattern was photographed using an SLR camera (3200 ASA black and white film) with exposure times of 1-4 ms to stop the motion of the fluid. Visualizations shown here extend roughly $\pm 30 \mathrm{~mm}$ from the center of the tank.

\section{Laser Velocimetry}

The laser velocimeter was identical to the fixed channel arrangement used by Parthasarathy and Faeth ${ }^{1}$ and will be described only briefly. A dual-beam forward-scatter configuration was used which had a measuring volume diameter and length of 0.1 and $1.2 \mathrm{~mm}$, respectively. Directional bias and ambiguity were eliminated using a Bragg-cell frequency shifter. The bath liquid was seeded with titanium dioxide particles ( $2.8 \mu \mathrm{m}$ nominal diameter) to provide data rates in the range $0.5-2 \mathrm{kHz}$. The velocities were found from the lowpass filtered analog output of a burst-counter signal processor, using a 12-bit analog/digital converter operating at a constant sampling frequency $(1-4 \mathrm{kHz})$, and storing $120 \mathrm{k}$ samples per traverse.

The laser velocimeter measuring location was fixed; therefore, the trajectory of the sphere was traversed to observe various points in the flow. The streamwise traverse was carried out by the motion of the sphere itself, with distances known from the position indicator of the linear positioner. Positioning accuracy in the streamwise direction was controlled primarily by uncertainties of locating the center of the sphere at the measuring volume and the time between samples, yielding an uncertainty of $0.4 \mathrm{~mm}$ for each velocity determination used during ensemble averaging. Cross-stream traverses were carried out by moving the wire support points with a manual traversing system attached to the struts, yielding a positioning accuracy of $0.2 \mathrm{~mm}$.

Measurements were carried out by ensemble averaging results from 20 to 120 traverses at a particular radial position and sphere Reynolds number. The number of traverses was selected to control the experimental uncertainties and was large in regions where effects of vortex shedding were important (due to large traverse-to-traverse variations of flow properties) and when rms velocity fluctuations were sought. Traverses only were initiated after bath disturbances from the previous traverse were sufficiently decayed (specified to be $\bar{u}^{\prime} / U_{s}<0.5 \%$ ). 
Table 1 Test conditions a

\begin{tabular}{rrrrrrr}
\hline $\begin{array}{l}R e \\
(-)\end{array}$ & $\begin{array}{c}\text { Glycerin } \\
\text { concentration }\end{array}$ & $\nu, \mathrm{mm}^{2} / \mathrm{s}$ & \multicolumn{1}{c}{$\begin{array}{c}C_{s}, \mathrm{~mm} / \mathrm{s} \\
(-)\end{array}$} & $\begin{array}{c}0, \mathrm{~mm} \\
\left(U_{o}\right)_{t} / U_{s} \mathrm{f}\end{array}$ \\
\hline 35 & 81 & 42.0 & 147 & $1.91^{\mathrm{c}}$ & 4.88 & 1.9 \\
60 & 81 & 42.0 & 252 & $1.42^{\mathrm{c}}$ & 4.22 & 2.0 \\
90 & 81 & $37.8-41.0$ & $340-369$ & $1.16^{\mathrm{c}}$ & 3.81 & 2.3 \\
170 & 76 & $24.0-28.0$ & $408-476$ & $0.86^{\mathrm{c}}$ & 3.29 & 2.6 \\
280 & 62 & $10.1-11.7$ & $283-328$ & $0.59^{\mathrm{d}}$ & 2.72 & 2.4 \\
400 & 62 & 10.2 & 408 & $0.603^{\mathrm{c}}$ & 2.75 & 2.0 \\
960 & 50 & 4.8 & 461 & $0.430^{\mathrm{c}}$ & 2.32 & 0.9 \\
4000 & 0 & 1.02 & 408 & $0.381^{\mathrm{e}}$ & 2.18 & 0.8 \\
\hline \hline
\end{tabular}

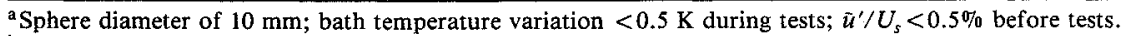

b Percent glycerin in water by mass.

${ }^{c} C_{D}=24\left(1+R e^{2 / 3} / 6\right) / R e$, from Putnam. ${ }^{28}$

dFitted from measured radial mean velocity distribution.

${ }^{\mathrm{e}} C_{D}=0.28+6 / R e^{1 / 2}+21 / R e$, from Kürten et al. ${ }^{29}$

${ }^{f}$ Based on a fixed virtual origin at $x_{o} / \theta=1$.

Experimental uncertainties varied with sphere Reynolds number and position in the wake, with measurements in the far wake terminated when background disturbances became significant in comparison to wake velocities. For data reported here, uncertainties ( $95 \%$ confidence) of mean streamwise velocities were less than $13 \%$, and those of rms streamwise velocity fluctuations were less than $25 \%$. All measurements were repeatable within these ranges over a period of testing of several months. These uncertainties are high in comparison to conventional laser velocimeter measurements due to the relatively low velocities and the ensemble averaging technique of the present tests.

\section{Test Conditions}

Test conditions are summarized in Table 1. Here and in the following, all velocities are relative to an inertial reference frame. The densities and viscosities of the test liquids were measured periodically during testing with a hydrometer and a Cannon/Fenske viscometer. Effects of bath temperature changes on kinematic viscosities were compensated by adjusting the sphere velocity to obtain the desired sphere Reynolds number.

Drag coefficients for the various sphere Reynolds numbers generally were computed from correlations due to Putnam ${ }^{28}$ and Kürten et al. ${ }^{29}$ The one exception was $R e=280$, which was at the onset of vortex shedding where existing drag correlations were suspect; therefore, $C_{D}$ at this condition was found from the measured velocity defect in the wake. Values of the initial momentum thickness of the wakes then were computed as follows ${ }^{21}$ :

$$
\theta=\left(C_{D} d^{2} / 8\right)^{1 / 2}
$$

\section{Wake Similarity}

\section{Turbulent Wake}

It is well known that mean velocity distributions in high Reynolds-number axisymmetric turbulent wakes become selfpreserving far from the drag-producing object. Within the self-preserving region, mean velocities correlate reasonably well with predictions based on a constant eddy viscosity over the wake cross section, in spite of the crudeness of this approximation; see Uberoi and Freymuth ${ }^{19}$ and references cited therein. Based on results appearing in Tennekes and Lumley, ${ }^{21}$ this yields the following expression for streamwise mean velocities $^{1}$ :

$$
\bar{u} / U_{s}=2.23\left[\left(x-x_{o}\right) / \theta\right]^{-2 / 3} \exp \left(-r^{2} / 2 \ell^{2}\right)
$$

where the characteristic width of the wake is given by

$$
\ell / \theta=0.47\left[\left(x-x_{o}\right) / \theta\right]^{1 / 3}
$$

and $x_{o}$ is the virtual origin of the flow: The local wake Reynolds number based on the centerline velocity and characteristic wake width then becomes

$$
R e_{w}=\ell \bar{u}_{c} / \nu=1.048\left(\theta U_{s} / \nu\right)\left[\left(x-x_{o}\right) / \theta\right]^{-1 / 3}
$$

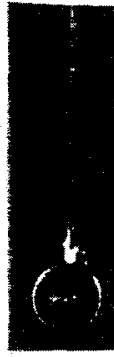

Re $=40$

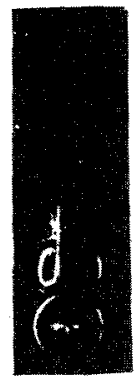

$\mathrm{Re}=200$

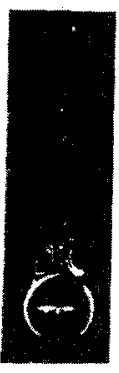

$\operatorname{Re}=60$

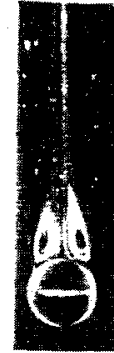

Re $=90$

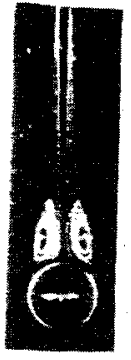

$\operatorname{Re}=120$

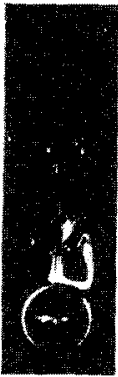

Re $=240$

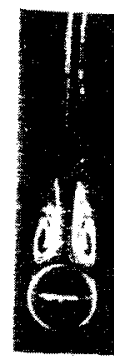

$R e=150$
Fig. 2 Visualization of near wakes for intermediate Reynolds numbers $(R e=40-280)$.

\section{Laminar Wake}

Laminar wake regions also were observed during the experiments. Streamwise mean velocities in this region are given by classical similarity analysis, as follows ${ }^{23}$ :

$$
\bar{u} / U_{s}=\left[\theta^{2} U_{s} / 4 \nu\left(x-x_{o}\right)\right] \exp \left(-r^{2} / 2 \ell^{2}\right)
$$

where

$$
\ell / \theta=\left[2 \nu\left(x-x_{o}\right) / \theta^{2} U_{s}\right]^{1 / 2}
$$

From Eqs. (5) and (6), the local wake Reynolds number based on the centerline velocity and the characteristic width in the laminar wake regime becomes

$$
\operatorname{Re}_{w}=\ell \bar{u}_{c} / \nu=\theta^{2}\left[U_{s}^{3} / 8 \nu^{3}\left(x-x_{o}\right)\right]^{1 / 2}
$$

The mean velocity $\bar{u}$ has been used in Eqs. (5-7), even for a steady laminar flow, because present values were obtained as ensemble averages which were influenced to some extent by background disturbances in the bath.

\section{Results and Discussion}

\section{Near- and Fast-Decay Wake Regions}

Photographs of the light-sheet illuminated dye traces for sphere Reynolds numbers in the range 40-280 appear in Fig. 2. 
This range is representative of conditions where vortex shedding is not present, $R e \leq 240$, up to conditions where vortex shedding just begins, $R e \approx 280$. In the region where they overlap $(R e<200)$, present observations are very similar to those Nakamura ${ }^{15}$ for freely falling spheres, except for minor effects due to different methods of introducing the dye. This suggests that the present horizontal wire support did not have a significant effect on flow properties near the sphere. For $20 \leq R e<200$ (the former being the lowest value considered), a stable and symmetric recirculation zone is attached to the downstream side of the sphere, with the size of the recirculation zone progressively increasing as $R e$ increases. Within this region, present measurements of the angle between the attachment points of the recirculation region were in good agreement with measurements for freely falling spheres due to Nakamura. ${ }^{15}$ Throughout this region, dye traces leaving the downstream end of the recirculation zone were smooth and gave little evidence of unsteady or turbulent-like behavior over the region where they could be seen. For $20 \leq R e \leq 240$, the recirculation zone still remained attached and stable; however, it generally was no longer symmetric. Additionally, the dye trace leaving the recirculation zone remained along the axis and was only slightly more irregular than for $R e<200$. As is evident from Fig. 2, however, this behavior changed significantly at $R e=280$ where vortex shedding began. Typical of behavior at higher $R e$, where effects of vortex shedding on wake properties were very significant, the recirculation zone was very unsymmetric and the dye trace leaves the recirculation zone near its edge.

As noted earlier, the wake flow changed at $R e \approx 280$ so that the recirculation zone was no longer stable and vortex shedding began. This observation is in good agreement with results for freely falling spheres, suggesting small effects of the wire support on the vortex-shedding process. For example, Magarvey and MacLatchy ${ }^{8}$ and Goldberg and Florsheim ${ }^{10}$ report $R e=300$ and 270 , respectively, for this transition for freely falling spheres. Dye traces at higher Reynolds numbers

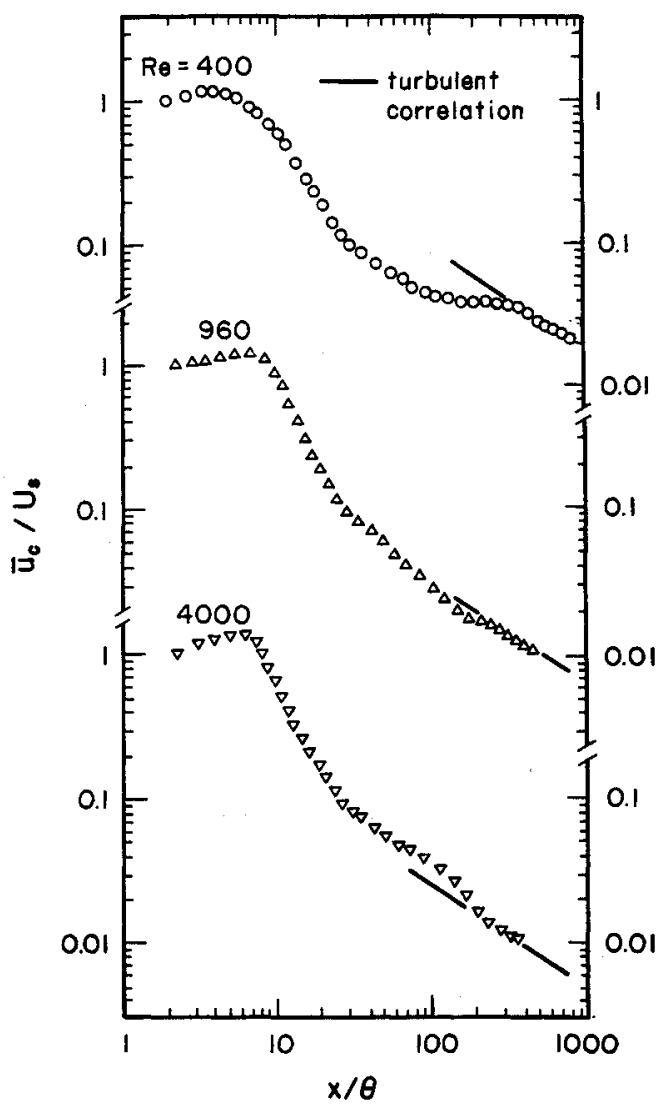

Fig. 3 Mean streamwise velocities along the wake axis $(R e=400$ 4000).

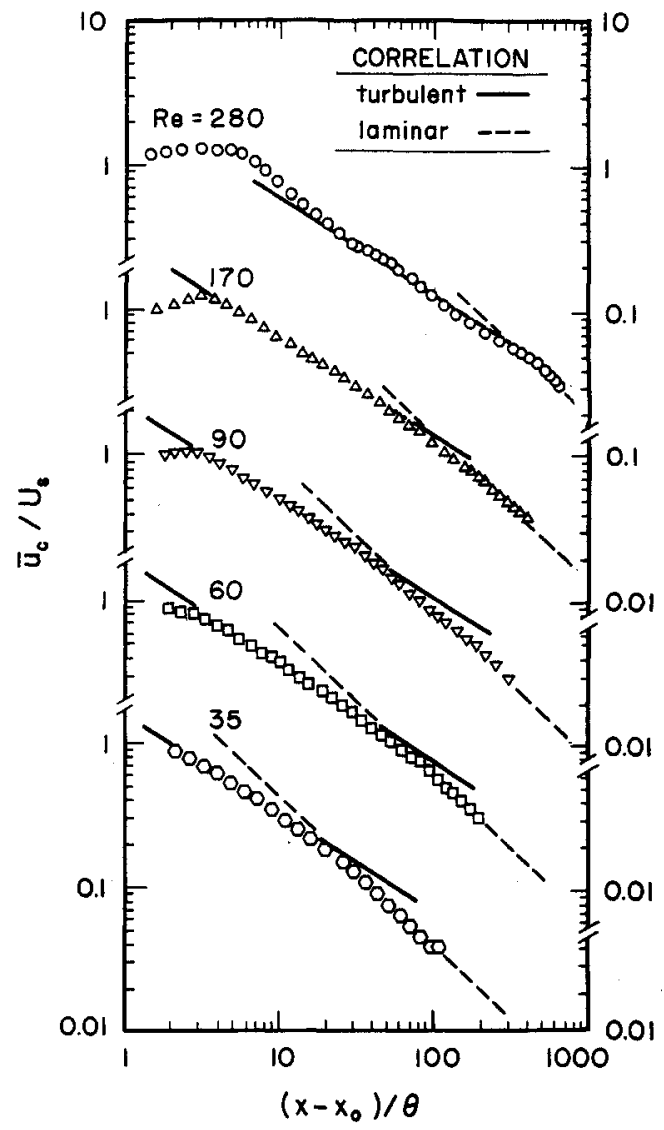

Fig. 4 Mean streamwise velocities along the wake axis $(\operatorname{Re}=35-280)$.

were similar to the observations of others, ${ }^{10,16}$ with increasing sphere Reynolds numbers yielding dye traces having finer scaled features and a large-scale back and forth distortion of the dye traces that is consistent with the double helix vortex tube structure thought to represent the vortex-shedding process. ${ }^{16}$

Streamwise mean velocities along the wake axis are illustrated in Fig. 3 for conditions where vortex shedding has a strong effect on mean velocities $(R e=400,960$, and 4000) and in Fig. 4 for conditions where effects of vortex shedding are either weak $(R e=280)$ or absent $(R e=35,60,90$, and 170). The results are plotted as a function of $x / \theta$ [or $\left(x-x_{o}\right) / \theta$ with $x_{o} / \theta=1$ as described later], so that they can be related to the similarity expressions for turbulent and laminar wakes, Eqs. (2) and (5). The measurements on the plots begin close to the rear stagnation point of the sphere, $x / d$ in the range $0.5-1.0$, and are ended when wake velocities become too low for accurate measurements.

The first feature evident from the results illustrated in Figs. 3 and 4 is the increase of velocity with increasing distance very near the sphere. This behavior is caused by deceleration of the backflow along the axis of the recirculation zone as the sphere is approached. The maximum velocity along the axis, however, is reached relatively close to the sphere, at $\left(x-x_{0}\right) / \theta$ of 4-6 or $x / d=1.4 \pm 0.2$, with the maximum tending to approach the sphere when the recirculation zone becomes smaller as $R e$ decreases.

When vortex shedding is present, $R e \geq 280$, mean velocities along the axis initially exhibit a more rapid decay than farther into the wake (cf. Figs. 3 and 4). The rate of decay in this region tends to decrease as $R e$ decreases toward the onset of vortex shedding. For example, if $\bar{u} / U_{s} \sim x^{-a}$ in the fast-decay wake region, maximum values of $a$ are $1.8,1.7,1.6$, and 1.2 for $R e=4000,960,400$, and 280 , respectively. Thus, the strength of the vorticity being shed from the sphere affects the enhancement of near-wake mixing rates. 


\section{Turbulent Wake Region}

The end of the fast-decay wake, and regions behaving like turbulent and laminar wakes, were identified using the classical similarity expressions of Eqs. (2) and (5). It was found that laminar wake behavior was in good agreement with Eq. (5), after choosing $x_{o} / \theta=1$; therefore, these correlations are entered directly for $R e$ in the range 35-280 where laminar wakes were observed (Fig. 4). Additionally, the turbulent portions of the wakes were in fair agreement with Eq. (2) using the same value of $x_{o} / \theta$ when eddy shedding was absent; however, the plots were offset when eddy shedding was present due to the influence of the fast-decay wake region on the virtual origin. Thus, to display results for the various wake regimes, with no change of virtual origin, a generalized correlation for the turbulent wake region was developed from Eqs. (2) and (3) as follows:

$$
\bar{u} / U_{s}=\left(U_{o}\right)_{t} / U_{s}\left[\left(x-x_{o}\right) / \theta\right]^{-2 / 3} \exp \left(-r^{2} / 2 \ell_{t}^{2}\right)
$$

where

$$
\ell_{t} / \theta=\left[U_{s} / 2\left(U_{o}\right)_{t}\right]^{1 / 2}\left[\left(x-x_{o}\right) / \theta\right]^{1 / 3}
$$

The values of the parameter $\left(U_{o}\right)_{t} / U_{s}$ were selected to provide the turbulent wake fits illustrated in Figs. 3 and 4 and are summarized in Table 1 . The parameter $\left(U_{o}\right)_{t} / U_{s}=2.23$ for exact agreement with Eq. (2): present measurements agree with this estimate for $R e \leq 400$, with an average value and standard deviation of 2.2 and 0.3. Results at $R e=960$ and 4000 exhibit lower values of $\left(U_{o}\right)_{t} / U_{s}$, near unity, which are consistent with the value of 0.9 found for the turbulent wake of a sphere at $R e=8600 .^{19}$

The results plotted in Fig. 3 indicate that the turbulent wake region is reached at $x / \theta \approx 200$ for $R e \geq 960$, which corresponds to $x / d$ in the range 40-50. This is comparable to other observations of the onset of fully developed turbulent wake properties for spheres and other blunt objects for $R e>1000$ (which involve vortex shedding from the object). ${ }^{17-19}$ In contrast, when vortex shedding is absent, Fig. 4 for $R e \leq 170$, the onset of the turbulent wake region is reached at $\left(x-x_{o}\right) / \theta \leq 4$, which corresponds to $x / d \leq 1.7-2.5$; this is comparable to Chevray's ${ }^{20}$ observation of a rapidly developing turbulent wake behind a slender spheroid at $R e=458,000$ (based on the body diameter) for conditions where vortex shedding also is absent. Chevray ${ }^{20}$ also points out that his behavior agrees with Townsend's ${ }^{30}$ prediction that the self-preserving turbulent wake should develop rapidly if production of turbulence within the separation region is small, i.e., stronger turbulence production in the presence of vortex shedding both enhances mixing rates in the fast-decay wake and causes the onset of the turbulent wake region to be deferred. Conditions at $R e=400$ and 280 (Figs. 3

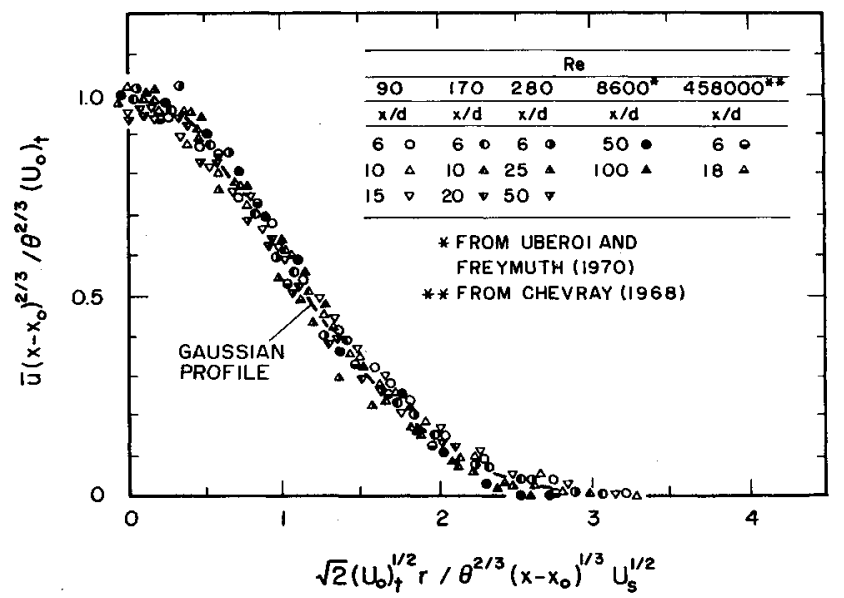

Fig. 5 Correlation of radial profiles of mean streamwise velocities in the turbulent wake region. and 4) represent intermediate behavior as the onset of vortex shedding is approached; at $R e=400$, onset of the turbulent wake region occurs near $x / \theta=300$ or $x / d \approx 80$ while at $R e=280$, onset occurs near $x / \theta=20$ or $x / d \approx 5$. It is not surprising that $x / d$ for onset of the turbulent wake becomes smaller as the onset of vortex shedding is approached; however, additional study is needed to establish that different relative rates of decay of vortices shed from the sphere and development of turbulence yield a maximum in the streamwise distance required to develop a turbulent wake structure for $\operatorname{Re}<10^{3}$.

For $R e \leq 280$, the measurements extended far enough so that a laminar wake region scaling according to Eq. (5) was reached (see Fig. 4). It is seen that the transition from turbulent to laminar wake behavior occurs where the two similarity expressions for $\bar{u}_{c} / U_{s}$ cross Eq. (2) for the turbulent wake because $\left(U_{o}\right)_{t} / U_{s} \approx 2.23$ for this range of $\operatorname{Re}$ and Eq. (5) for the laminar wake. Thus, an expression for the transition condition can be obtained by equating Eqs. (2) and (5) to yield

$$
\left(x-x_{o}\right)_{\mathrm{tr}} / \theta=1.41 \times 10^{-3}\left(\theta U_{s} / \nu\right)^{3}
$$

where this expression only has been established for $R e<400$. Substituting Eq. (10) into either Eq. (4) or Eq. (7) yields a wake Reynolds number at transition from the turbulent to laminar wake regimes of $R e_{w \mathrm{tr}}=9.4$. Tennekes and Lumley ${ }^{21}$ note that this transition should take place for $R \boldsymbol{e}_{w t r}$ on the order of unity. This is not in disagreement with present findings in view of the somewhat arbitrary selection of velocity and length scales in the definition of $R e_{w}$ and the range of $\left(x-x_{o}\right) / \theta$ required to complete transition from turbulent to laminar wake behavior.

The radial profiles of mean streamwise velocities in the turbulent wake region are illustrated in Fig. 5. In addition to present measurements, results from Uberoi and Freymuth ${ }^{19}$ for a sphere having a $R e=8600$ and Chevray ${ }^{20}$ for a slender body having a $R e=458,000$ (which involve conditions where vortex shedding is present and absent, respectively) are shown on the plots. Variations of the virtual origin are handled by scaling in terms of $\left(U_{o}\right)_{t} / U_{s}$ through Eqs. (8) and (9) as before. The value of $\left(U_{o}\right)_{t} / U_{s}=0.9$ for the measurements of Uberoi and Freymuth, 19 which is comparable to present results when effects of vortex shedding are strong, as noted earlier. The higher value of $\left(U_{o}\right)_{t} / U_{s}=3.0$ for the measurements of Chevray $^{20}$ is typical of present measurements when vortex shedding is either absent or weak; see Table 1.

All of the results illustrated in Fig. 5 are seen to be reasonably correlated with each other and with the Gaussian velocity distribution function found from simplified similarity theory for the self-preserving turbulent wake. ${ }^{21}$ Present measurements are identified by $x / d$ but they extend over the full range where $\bar{u}_{c} / U_{s}$ agrees with turbulent wake scaling in Fig. 4 . Thus, results illustrated in Fig. 5 extend from $R e_{w}>90$ for measurements from Refs. 19 and 20 down to $R e_{w} \approx 10$ near transition to laminar wake behavior for the present measurements, with effects of vortex shedding both present and absent. Clearly, mean velocity distributions within turbulent wakes generally are independent of these factors, after appropriate definition of a virtual origin or a velocity scale like $\left(U_{o}\right)_{t}$.

The relative insensitivity of mean velocities within turbulent wakes to the specific properties of the turbulence are highlighted by considering streamwise rms velocity fluctuations along the axis. These results are plotted in terms of the turbulence intensity at the axis $\left(\bar{u}^{\prime} / \bar{u}\right)_{c}$ as a function of $x / d$ in Fig. 6. The measurements of Uberoi and Freymuth ${ }^{19}$ are shown in the figure, along with present measurements for $R e$ in the range 280-4000 (present measurements were not feasible at lower values of $R e$ because excessive numbers of traverses were required to establish reliable values of $\bar{u}_{c}^{\prime}$ ). Results for the full range of $x / d$ of the measurements are illustrated with the portion in the turbulent wake region (or the fully developed turbulent wake region identified in Ref. 19) denoted by solid symbols. These results have not been corrected for background disturbances due to uncertainties about these effects in 


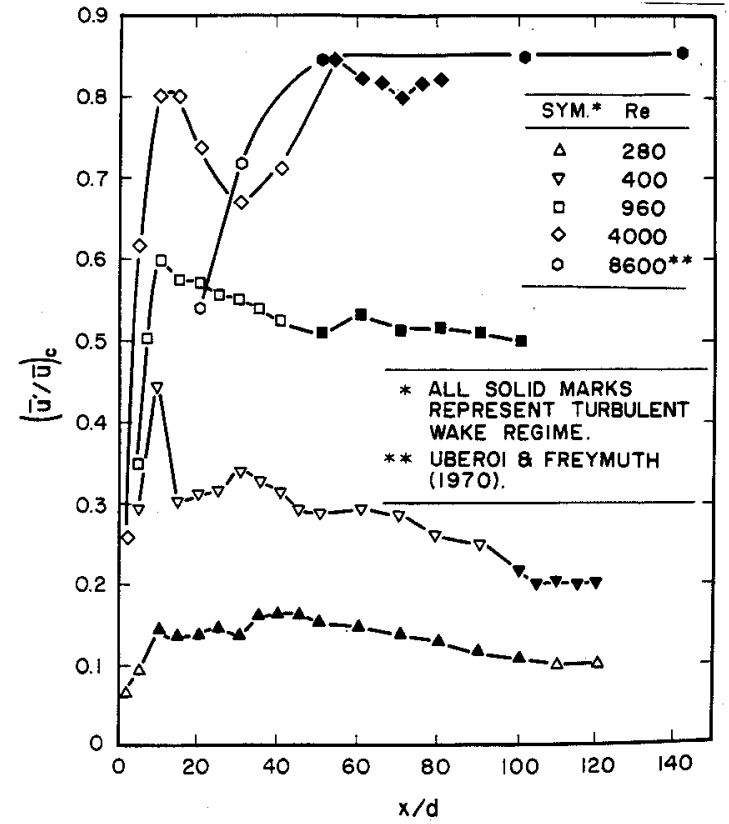

Fig. 6 Streamwise rms velocity fluctuations along the wake axis (Re $=\mathbf{2 8 0}-\mathbf{8 6 0 0}$ ).

the strongly directed near-wake region. Outside this region, the corrections are small except for the latter parts of the wakes for $R e \leq 960$; corrected results for the turbulent wakes will be considered subsequently.

Present measurements of $\left(\bar{u}^{\prime} / \bar{u}\right)_{c}$ within the turbulent wake region at $R e=4000$ are in reasonably good agreement with Uberoi and Freymuth ${ }^{19}$ in Fig. 6: turbulence intensities are nearly constant at roughly $85 \%$ and the onset of this regime is in the range $x / d=40-60$. Thus, within this regime, $\bar{u}_{c}^{\prime}$ $\sim\left(x-x_{0}\right)^{-2 / 3}$ from Eq. (2) as observed during a number of past studies. For $R e \leq 960$, however, turbulence intensities in the turbulent wake region progressively decrease with decreasing $R e$; nevertheless, even though these turbulence intensities are low in comparison to high Reynolds-number turbulent wakes, they still are comparable to values found in the actively turbulent flows, e.g., near the axis of turbulent jets and pipe flows. ${ }^{21,22}$ Additionally, at these lower Reynolds numbers, there is a tendency for the turbulence intensity to decrease with increasing distance; this is particularly evident beyond the initial development region for $R e=280$ (i.e., $x / d \geq 40$ ) where present measurements captured an extended turbulent wake region. Such behavior is expected as the turbulent wake decays toward transition to the laminar wake region and will be considered in more detail later.

Effects of vortex shedding on turbulence intensities in the near wake region also can be seen from the results plotted in Fig. 6. For present measurements at $R e=4000$, the turbulence intensity reaches a peak near $x / d=10$, due to strong effects of vortex shedding, and then decreases as the vortex pattern decays; subsequently, the development of wake turbulence causes the turbulence intensity to increase again and finally become nearly constant in the self-preserving wake region (this latter portion corresponding to behavior seen by Uberoi and Freymuth ${ }^{19}$ at comparable $R e$ ). With strong effects of vortex shedding at low $R e$, e.g., $R e=960$ and 40 , the peak in turbulence intensity due to vortex shedding still is evident near the sphere; however, the intensity subsequently decays monotonically to conditions in the turbulent wake region. For $R e=40$, the maximum turbulence intensity due to vortex shedding is largest in comparison to values in the turbulent wake region, roughly 2.5 times larger; thus, the greater decay required to reach turbulent wake conditions probably accounts for the larger $x / d$ required to reach self-preserving turbulent wake conditions, as noted earlier. Finally, when vortex shedding is weak $(R e=280)$ there is no peak in the turbulence intensity prior to the turbulent wake region and the onset of this region (with respect to the mean velocity distribution) occurs close to the sphere. However, turbulence still develops near the sphere, reaching a maximum intensity near $x / d=40$, which is representative of the development region for high Reynolds-number turbulent wakes. ${ }^{19}$

The character of the decay of turbulence during approach to transition from turbulent to laminar wake behavior (with respect to mean velocities) is illustrated in Fig. 7. Present data on this figure has been corrected for ambient disturbances in the usual manner, i.e., $\bar{u}_{c}^{\prime 2}=\bar{u}_{c m}^{\prime 2}-\bar{u}_{\infty}^{\prime 2}$, where $\bar{u}_{c m}^{\prime 2}$ is the initial measured value and $\bar{u}_{\infty}^{\prime 2}$ is the bath disturbance level prior to traversing the sphere. The results involve turbulence intensity at the wake axis plotted as a function of the local wake Reynolds number. The findings of Carmody ${ }^{17}$ and Uberoi and Freymuth ${ }^{19}$ a high wake Reynolds numbers are shown on the plot along with present results at moderate wake Reynolds numbers. These measurements involve fully developed turbulent wake conditions observed at $x / d \geq 40$ for Uberoi and Freymuth ${ }^{19}$ and present tests, and at $x / d=15$ for the high Reynolds-number results of Carmody. ${ }^{17}$

Taken together, the results illustrated in Fig. 7 suggest a reasonable correlation of wake turbulence properties in terms of the wake Reynolds number. For $R e_{w}>70$, turbulence intensities along the axis are nearly constant with $\bar{u}_{c}^{\prime} / \bar{u}_{c}$ in the range 0.85-0.92. At lower wake Reynolds numbers, however, turbulence intensities rapidly decrease (in terms of $R e_{w}$ ) in the final decay period as conditions for transition to a laminar wake are approached. Several proposals for the decay of turbulence energy in the final decay period have been made ${ }^{24-27}$; present results are in reasonable agreement with estimates for axisymmetric wakes, ${ }^{25,27}$ where $\bar{u}^{\prime 2} \sim t^{-5 / 2}$. Noting that $x \sim U_{s} t$ for the fully developed turbulent wake region $(x / d \geq 40)$ then yields the following relationship between turbulence intensity and local wake Reynolds number, using Eqs. (2) and (4):

$$
\left(\bar{u}^{\prime} / \bar{u}\right)_{c}=C R e_{w}^{7 / 4}
$$

The best fit of present data $\left(10<R e_{w}<30\right)$ yields $C=1.3$ $\times 10^{-3}$ with a standard deviation of $4 \times 10^{-4}$.

The best-fit correlation of Eq. (11) is plotted in Fig. 7, where it is seen to provide a reasonable fit of present measurements for various values of $R e$ and $10 \leq R e_{w} \leq 30$. If the same decay law was adopted from the laminar wake region, Eqs. (5) and (7) yield $(\bar{u})_{c} \sim R e_{w}^{1 / 2}$, which implies a much slower decay rate in terms of $R \boldsymbol{e}_{w}$ for laminar than turbulent wakes. Unfortunately, experimental evaluation of the transition of velocity fluctuations from turbulent to laminar wake behavior was not possible due to limitations of experimental uncertainties. Finally,

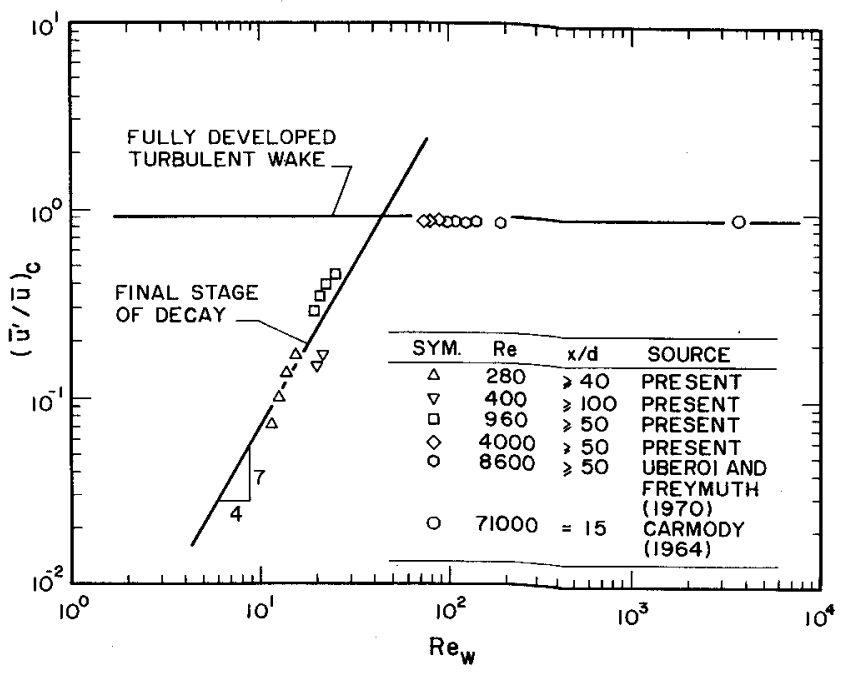

Fig. 7 Streamwise turbulence intensities along the axis as a function of wake Reynolds number in the turbulence wake region $(R e=280$ $\mathbf{- 7 1 , 0 0 0 ) \text { . }}$ 


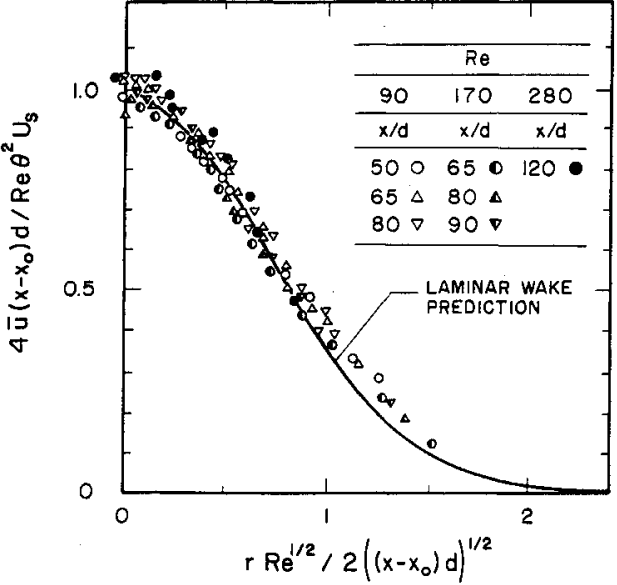

Fig. 8 Correlation of radial profiles of mean streamwise velocities in the laminar wake region $(\operatorname{Re}=90-280)$.

the alternative final decay behavior proposed by Tan and Ling, ${ }^{26} \bar{u}^{\prime 2} \sim t^{-2}$, yields $\left(\bar{u}^{\prime} / \bar{u}\right)_{c} \sim R e_{w}^{o}$ and $R e_{w}$ in the laminar and turbulent wake regions, the latter implying a much slower rate of decay than present observations.

\section{Laminar Wake Region}

The radial profiles of mean streamwise velocities in the laminar wake region are illustrated in Fig. 8. Results are shown for $R e=90,170$, and 280 for $x / d$ in the range $50-120$, plotted according to the similarity relationships of Eqs. (5) and (6) with $x_{o} / \theta=1$. The measurements do not extend to the edge of the flow because low velocities in this region precluded results within the experimental uncertainties specified earlier. Over the range considered, however, the measurements agree with Eqs. (5) and (6) within experimental uncertainties in spite of the relatively high turbulence levels and the slow rate of decay of turbulence. An explanation for this behavior is that turbulence in the final decay period is not connected over the flow cross section similar to high Reynolds-number turbulent wakes; instead, it involves noninteracting localized regions of decaying turbulence (called turbulence spots, stratified turbulence, or dilute vortex streaks). ${ }^{24-27}$ The absence of connectedness prevents mixing (entrainment or engulfment of ambient fluid) by large-scale turbulence structures that extend over a significant portion of the width of the wake, characteristic of high Reynolds-number turbulent wakes. Then, the overall mixing is dominated by laminar viscous effects so that mean velocity distributions adjust accordingly with the decaying turbulence spots only mildly affecting flow properties.

\section{Conclusions}

The major conclusions of the study are as follows:

1) The properties of the recirculation zone on the downstream side of the sphere were similar to earlier observations $7-16$ : the recirculation zone was stable and symmetric for $R e \leq 200$, stable and unsymmetric for $200<R e<280$, and unstable with vortex shedding present for $R e \geq 280$.

2) The wakes exhibited three regions: a fast-decay wake region near the sphere that only was observed when vortex shedding was present, followed in succession by turbulent and laminar wake regions.

3) Mean velocities within the turbulent wake region scaled according to similarity predictions for self-preserving turbulent wakes, Eqs. (2), (3) or (8), (9), even though turbulence intensities along the axis varied in the range $10-85 \%$ and vortex shedding was present or absent. The main effect of vortex shedding on the turbulent wake region was to defer its onset, by an order of magnitude, due to the presence of the fast-decay wake region.

4) Transition from the turbulent to the laminar wake region occurred where similarity estimates of mean streamwise veloc- ities along the axes for laminar and turbulent wakes were the same, Eq. (10). This corresponded to $R e_{w} \approx 10$.

5) Within the fully developed turbulent wake region $(x / d$ $\geq 40$ ), $\left(\bar{u}^{\prime} / \bar{u}\right)_{c} \approx 85 \%$ for $R e_{w}>70$, typical of high Reynoldsnumber axisymmetric wakes; however, as conditions for transition to laminar wakes were approached, $\left(\bar{u}^{\prime} / \bar{u}\right)_{c} \sim R e_{w}^{7 / 4}$, which is consistent with the final decay period of turbulence in axisymmetric wakes. ${ }^{25,27}$

6) Even though velocity fluctuations in the laminar wake region were significant, mean velocity distributions scaled according to laminar similarity predictions, Eqs. (6) and (7). This is plausible because turbulence in the final decay period involves isolated turbulent spots so that overall mixing is not controlled by the connected large-scale structures found in turbulent wakes; instead, mixing is dominated by laminar viscous effects and the mean velocity distributions adjust accordingly.

\section{Acknowledgments}

This research was sponsored by the Air Force Office of Scientific Research, Air Force Systems Command, USAF, under Grant AFOSR-89-0516. The U.S. Government is authorized to reproduce and distribute reprints for Governmental purpose notwithstanding any copyright notation thereon.

\section{References}

${ }^{1}$ Parthasarathy, R. N., and Faeth, G. M., "Turbulence Modulation in Homogeneous Dilute Particle-Laden Flows," Journal of Fluid Mechanics, Vol. 220, Nov. 1990, pp. 485-514.

2Mizukami, M., Parthasarathy, R. N., and Faeth, G. M., "Particle-Generated Turbulence in Homogeneous Dilute Dispersed Flows," International Journal of Multiphase Flow, Vol. 18, No. 3, May 1992, pp. 397-412.

${ }^{3}$ Torobin, L. B., and Gauvin, W. H., "Fundamental Aspects of Solids-Gas Flow, Part I: Introductory Concepts and Idealized Sphere Motion in Viscous Regime," Canadian Journal of Chemical Engineering, Vol. 37, No. 4, Aug. 1959, pp. 129-141; also "Part II: The Sphere Wake in Steady Laminar Fluids," Vol. 37, No. 5, Oct. 1959, pp. 167-176; also "Part V: The Effects of Fluid Turbulence on the Particle Drag Coefficient," Vol. 38, No. 6, Dec. 1960, pp. 189-200.

${ }^{4}$ Pruppacher, H. R., LeClair, B. P., and Hamielec, A. E., "Some Relations Between Drag and Flow Pattern of Viscous Flow Past a Sphere and a Cylinder at Low and Intermediate Reynolds Numbers," Journal of Fluid Mechanics, Vol. 44, Pt. 4, Dec. 1970, pp. 781-790.

${ }^{5}$ Rimon, Y., and Cheng, S. I., "Numerical Solution of a Uniform Flow Over a Sphere at Intermediate Reynolds Numbers," Physics of Fluids, Vol. 12, No. 5, 1969, pp. 949-959.

${ }^{6}$ Roos, F. W., and Willmarth, W. W., "Some Experimental Results on Sphere and Disk Drag," AIAA Journal, Vol. 9, No. 2, 1971, pp. 285-291.

${ }^{7}$ Magarvey, R. H., and Bishop, R. L., "Wakes in Liquid-Liquid Systems," Physics of Fluids, Vol. 4, No. 7, 1961, pp. 800-805.

${ }^{8}$ Magarvey, R. H., and MacLatchy, C. S., "Vortices in Sphere Wakes," Canadian Journal of Physics, Vol. 43, Sept. 1965, pp. 16491656.

${ }^{9}$ Willmarth, W. W., Hawk, N. E., and Harvey, R. L., "Steady and Unsteady Motions and Wakes of Freely Falling Disks," Physics of Fluids, Vol. 7, No. 2, 1964, pp. 197-208.

${ }^{10}$ Goldberg, A., and Florsheim, B. H., "Transition and Strouhal Number for the Incompressible Wake of Various Bodies," Physics of Fluids, Vol. 9, No. 1, 1966, pp. 45-50.

${ }^{11}$ List, R., and Hand, M. J., "Wakes of Freely Falling Water Drops," Physics of Fluids, Vol. 14, No. 8, 1971, pp. 1648-1655.

${ }^{12}$ Viets, H., "Accelerating Sphere-Wake Interaction," AIAA Journal, Vol. 9, No. 10, 1971, pp. 2087-2089.

${ }^{13}$ Calvert, J. R., "Some Experiments on Flow Past a Sphere," Aeronautical Journal of the Royal Aeronautical Society, Vol. 76, April 1972, pp. 248-250.

${ }^{14}$ Achenbach, E., "Vortex Shedding from Spheres," Journal of Fluid Mechanics, Vol. 62, Pt. 2, Jan. 1974, pp. 209-221.

${ }^{15}$ Nakamura, I., "Steady Wake Behind a Sphere," Physics of Fluids, Vol. 19, No. 1, 1976, pp. 5-8.

${ }^{16} \mathrm{Pao}, \mathrm{H} .-\mathrm{P}$. , and Kao, T. K., "Vortex Structure in the Wake of a Sphere,"' Physics of Fluids, Vol. 20, No. 2, 1977, pp. 187-191.

${ }^{17}$ Carmody, T., "Establishment of the Wake Behind a Disk," Journal of Basic Engineering, Vol. 87, No. 4, Dec. 1964, pp. 869-883.

${ }_{18}$ Hwang, N. H. C., and Baldwin, L. V., "Decay of Turbulence in Axisymmetric Wakes,' Journal of Basic Engineering, Vol. 88, No. 1, 
March 1966, pp. 261-268.

${ }^{19}$ Uberoi, M. S., and Freymuth, P., "Turbulent Energy Balance and Spectra of the Axisymmetric Wake," Physics of Fluids, Vol. 13, No. 9, 1970, pp. 2205-2210.

${ }^{20}$ Chevray, R., "The Turbulent Wake of a Body of Revolution," Journal of Basic Engineering, Vol. 90, No. 2, June 1968, pp. 275-284.

${ }^{21}$ Tennekes, H., and Lumley, J. L., $A$ First Course in Turbulence, MIT Press, Cambridge, MA, 1972, pp. 113-124.

${ }^{22}$ Hinze, J. O., Turbulence, 2nd ed., McGraw-Hill, New York, 1975, pp. 496-581.

${ }^{23}$ Schlichting, H., Boundary Layer Theory, 7th ed., McGraw-Hill, New York, 1977, pp. 234, 235, and 599.

${ }^{24}$ Batchelor, G. K., and Townsend, A. A., "Decay of Turbulence in the Final Period," Proceedings of the Royal Society (London), Vol. A194, No. 8, Nov. 1948, pp. 527-543.

${ }^{25}$ Phillips, O. M., "Final Period of Decay of Non-Homogeneous
Turbulence," Proceedings of the Cambridge Philosophical Society, Vol. 52, Pt. I, Jan. 1956, pp. 135-151.

${ }^{26}$ Tan, H. S., and Ling, S. C., "Final Stage of Decay of Grid-Produced Turbulence,'" Physics of Fluids, Vol. 6, No. 12, 1963, pp. 16931699.

${ }^{27}$ Lee, D. A., and Tan, H. S., "Study of Inhomogeneous Turbulence," Physics of Fluids, Vol. 10, No. 6, 1967, pp. 1224-1230.

${ }^{28}$ Putnam, A., "Integrable Form of Droplet Drag Coefficient," American Rocket Society Journal, Vol. 31, No. 10, 1961, pp. 1467, 1468.

${ }^{29}$ Kürten, H., Raasch, J., and Rumpf, H., "Beschleunigung eines Kugelförmen Feststoffteilchens im Strömungsfeld Konstanter Geschwindigkeit," Chemi-Ingenieur-Technik, Vol. 38, No. 9, Jan. 1966, pp. 941-948.

${ }^{30}$ Townsend, A. A., The Structure of Turbulent Shear Flow, 2nd ed., Cambridge Univ. Press, Cambridge, England, 1976, Chap. 6.

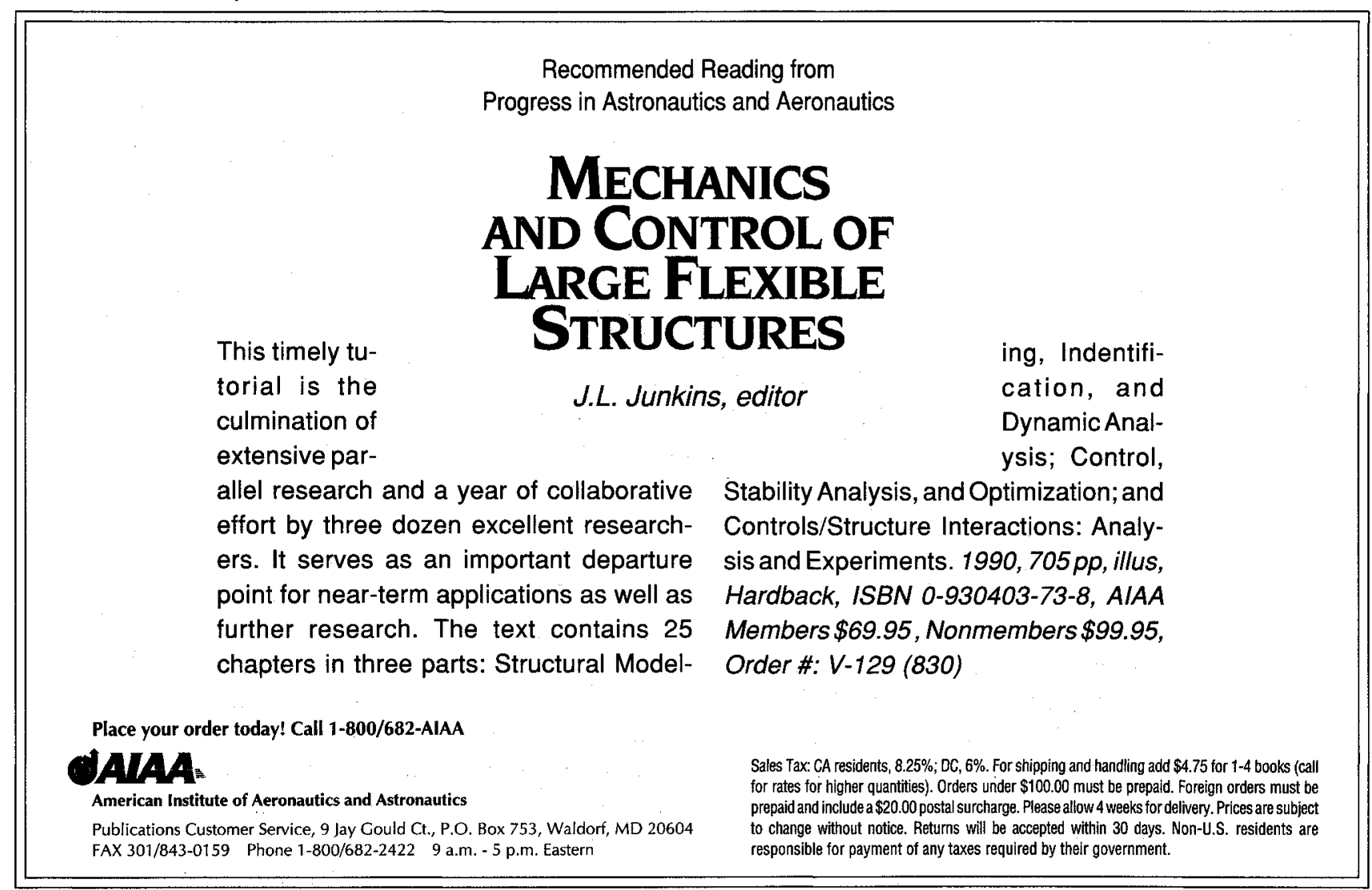

